Muro de la Investigación, 2018: 3(1), Enero-Julio, ISSN: 2523-2886

DOI:https://doi.org/10.17162/rmi.v3i1.1113

\title{
Programa "Jugando con los sonidos" y conciencia fonológica en niños de grado de transición de una institución educativa colombiana
}

\author{
Andrea Sánchez Pinzón ${ }^{1} y$ Gabriela Requena Cabral ${ }^{2 a}$ \\ Universidad Peruana Unión, Perú ${ }^{12}$
}

Recibido: 15 de julio de 2018

Aceptado: 22 de noviembre de 2018

\begin{abstract}
Resumen
El objetivo de este estudio fue comprobar la eficacia del programa "Jugando con los sonidos" para incrementar los niveles de la conciencia fonológica en niños del nivel de transición de una institución educativa pública colombiana. Participaron 48 estudiantes, en edad promedio de 6 años 2 meses; los niños fueron distribuidos al azar; 24 estudiantes en el grupo control y 24 estudiantes en el grupo experimental. El tipo de diseño fue cuasi - experimental. Todos los estudiantes fueron evaluados con el subtest de Conciencia Fonológica del Test de Habilidades Prelectoras de Velarde, Canoles, Meléndez y Ligán (2013). Al grupo experimental se le aplicó el programa Psicolingüístico de la conciencia fonológica a lo largo de cuatro meses y el grupo control continuo con su enseñanza regular. En los resultados obtenidos en el Post- Test se puede evidenciar un rango promedio superior en el grupo experimental en comparación al grupo control. Esto se reafirma en el momento de aplicar la prueba basada en el estadístico de U de Mann-Whitney dado que presenta un Valor $\mathrm{p}=0.000$ muy pequeño (menor que 0.01 ) Por lo tanto se puede evidencian la efectividad del programa para incrementar los niveles de la conciencia fonológica en estudiantes del nivel de transición. En conclusión, existe una diferencia significativa entre los resultados obtenidos en la prueba inicial y la prueba final, siendo mayores la prueba final del grupo experimental.
\end{abstract}

Palabras clave: conciencia fonológica, nivel de rimas, nivel de sílabas, nivel de fonemas

\author{
"Playing with sounds" program and phonological awareness \\ in children from a Colombian educational institution
}

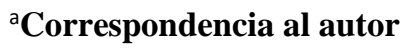

E-mail: grequenacabral@gmail.com
} 


\begin{abstract}
The objective of this study was to verify the effectiveness of the "Playing with sounds" program to increase the levels of phonological awareness in transition level children of a Public Educational Institution, where 48 students participated, with an average age of 6 years 2 months; the children were randomized; 24 students in the control group and 24 students in the experimental group. The type of design is Quasi - Experimental. All the students were evaluated with the subtest of Phonological Awareness of the Pre-reading Skills Test of Velarde, Canoles, Meléndez and Ligán (2013). The psycholinguistic program of phonological awareness was applied to the experimental group over four months and the control group continued with its regular teaching. In the results obtained in the Post-Test a higher average range can be seen in the experimental group compared to the control group. This is reaffirmed at the time of applying the test based on the Mann-Whitney $\mathrm{U}$ statistic since it has a very small $\mathrm{P}$ value $=.000$ (less than 0.01 ). Therefore, the effectiveness of the program to increase the levels of phonological awareness in students of the transition level is shown. In conclusion, there is a significant difference between the results obtained in the initial test and the final test, with the final test of the Experimental group being higher.
\end{abstract}

Key-words: Phonological Awareness, Rhyme Level, Syllable Level, Phoneme Level

\title{
Introducción
}

En las pruebas PISA (por sus siglas en inglés: Programe For International Student Assesment) 2012 se pudo evidenciar que Colombia, junto a otros países de latinoamérica ocuparon los últimos lugares de la lista. El objetivo de la OCDE con las pruebas PISA es comparar el desarrollo económico de los países con sus niveles de educación, donde encuentran una relación directa entre las dos dimensiones, En efecto los estudiantes con estrato socioeconómico bajo se desempeñan de forma inferior que los de nivel socioeconómico alto, además los estudiantes que no asistieron a ningún nivel del preescolar tuvieron menor desempeño que los que si asistieron (Bos, Gaminian y Veggentaa, 2014).

En relación con lo anterior Fernández (2002) afirma que la pobreza alcanza tres dimensiones esenciales de la vida humana: la longevidad, los conocimientos y un nivel de vida decoroso. En cuanto al conocimiento alude a la exclusión o niveles bajos del mundo de la lectura y la escritura Bedoya (2011) exdirector del ICFES (Instituto Colombiano para la Evaluación de la Educación) realizó un análisis estadístico de los resultados Saber Pro (2009, 2010) y muestra que entre más alto el nivel socioeconómico mayores puntajes obtienen.

También se plantea que los niños preescolares en estratos socioeconómicos bajos tienden a tener bajo desarrollo en las habilidades lingüísticas, menor vocabulario receptivo y expresivo y 
pobre conciencia fonológica, también resalta que no sólo se debe a los bajos niveles socioeconómicos sino también a la interacción padre-hijo, los factores ambientales en el hogar, el acceso a material impreso, la práctica de la lectura. Adams (Citado por Florez, Restrepo y Schwanenflugel, 2009).

Bustamante (2015) menciona que, aunque el Gobierno de Colombia se ha propuesto ser el país más educado de la región para el 2025, objetivo que busca mejorar el nivel educativo de los estudiantes, todavía no lo ha conseguido. Sus estudiantes de nivel primaria y secundaria no muestran avances significativos en las pruebas nacionales de lenguaje. Estos resultados se demuestran en las pruebas Saber $3 .^{\circ}, 5{ }^{\circ}$ y $9 .^{\circ}$, aplicadas por el ICFES.

Maya (2014) declara que, Medellín la ciudad más innovadora de Colombia con el lema "la más educada" continúa obteniendo bajos resultados en el área de lectura en las pruebas nacionales Saber. Teniendo en cuenta los anteriores argumentos a los bajos niveles de lectura y habilidades lingüísticas que presentan los niños en estratos socioeconómicos bajos se propone implementar medidas desde los primeros años escolares.

Todos los niños tienen los mismos derechos y oportunidades, independientemente del nivel socioeconómico. Es así que las maestras de preescolar poseen una tarea importante y trascendental de brindar con equidad a todos los niños, las bases necesarias para el aprendizaje de la lectura y escritura, habilidad que le permitirá avanzar en sus conocimientos y niveles educativos.

Teniendo en cuenta la importancia de estimular e intervenir la conciencia fonológica desde los primeros niveles de preescolar se han diseñados diversos programas internacionales como el de Orton-Gillinghan, Método Fernald, Lindamood Phoneme Sequecing Program y De Alfa Omega. En España se han publicado materiales como Programa de Habilidades metafonológicas, Lotos Fonéticos, Vamos a jugar con las palabras, las sílabas, los sonidos y las letras, y Aprendiendo a Leer, material de apoyo (Santiuste y López, 2004).

En Latinoamérica se han desarrollado diferentes estudios relacionados con la conciencia fonológica que demuestran su importancia para la adquisición del lenguaje oral y escrito (Velarde, 2008; Arancibia, Bizama y Sáenz, 2012; Benardi, Mishima y Dos Santo, 2013; Gallego, Angustias y Gómez, 2015; Rosenberg y Stein, 2015 y Gutiérrez y Díez, 2017).

El Programa "Jugando con los Sonidos" fue diseñado y validado en Perú por la doctora Ester Velarde Consoli en niños de 8 a 10 años en estrato socioeconómico bajo del Callao, donde 
el grupo experimental mejoró significativamente en todas las áreas de las tareas de la conciencia fonológica evaluadas y mejoras en la decodificación y comprensión lectora (Velarde, 2008).

Caman (2010) aplicó también en el Perú el programa "Jugando con los sonidos" a niños de la edad de 5 años, de estrato socioeconómico bajo, los resultados demostraron diferencias significativas mayor en todos los niveles de la conciencia fonológica después de ser evaluados. Concluyendo la efectividad del programa para niños de inicial.

En Colombia existen pocas investigaciones relacionadas con la conciencia fonológica, se evidencian trabajos de grados y unos cuantos artículos científicos, de los cuales se puede deducir que los niños de estrato socioeconómico bajos o retraso en el aprendizaje lector, después ser intervenido obtienen mayores niveles de conciencia fonológica y mejoran su capacidad lectora, demostrando una relación directa entre la conciencia fonológica y la lectura, también la importancia de ser estimulada desde los primeros años (Alsina, León y Pino, 2011; Meneses, Garzón, Macías, 2012; Cardona y Cadavid, 2013; Cadavid, Quijano, Tenorio y Rojas, 2014).

La conciencia fonológica es definida como un prerrequisito importante para el aprendizaje de la lectura y escritura, tal vez el más influyente, ya que es una habilidad que permite reconocer al lenguaje como divisible y reflexionar sobre la existencia de elementos discretos no significativos del lenguaje oral, y así manipular los sonidos de una emisión verbal (Ardila, Rosselli y Matute, 2005). Bravo (2003) la concibe como una habilidad Metalingüística, cuando los niños son conscientes que el lenguaje oral tiene componentes fonémicos, que se pueden aislar o añadir a su voluntad. El término metalingüística surgió de una serie de investigaciones dedicadas al estudio de la conciencia del lenguaje en los niños (Tunmer y Bowey citado por Carrillo y Gallego, 1992).

Estos conceptos apuntan a que la conciencia fonológica no es sólo una parte del lenguaje que permite transmitir significado a la comunicación lingüística de forma que pueda ser atendida por el oído, sino que la conciencia lingüística es de alguna pertenencia fonológica particular o de alguna parte constituyente. El objetivo de este estudio fue comprobar la eficacia del programa "Jugando con los sonidos" para incrementar los niveles de la conciencia fonológica en niños del nivel de transición de una institución educativa pública colombiana.

Según el modelo evolutivo, el desarrollo de la conciencia fonológica ocurre entre los 4 y 8 años de edad, que va desde la conciencia silábica hasta el dominio del fonema, surgiendo desde antes de la adquisición de la lectura y la Escritura hasta el dominio de la misma (Anthony y Francis, 2005). 
Según Rueda (1995) propone tres niveles de conocimiento fonológico enumerados de menor a mayor complejidad.

Conocimiento de la rima:

Los niños pueden adquirir esta habilidad antes del lenguaje escrito, debido a su facilidad para ser reconocida, ya que sólo requiere de una función perceptiva, fonológica y normalidad en el desarrollo del lenguaje, para establecer semejanzas y diferencias entre las palabras escuchadas (Guarneros y Vega, 2014).

Jager, Foorman, Lundberg y Beeler (1998) confirman que; este segmento no es tan indispensable para un conocimiento fonológico firme, ya que tan sólo es un paso para la dirección correcta, dando así más relevancia a la sílaba y al fonema.

\section{Conocimiento Silábico:}

La sílaba como la rima es una de las unidades más fácil que los niños pueden reconocer, y se presentan incluso antes de la lectura. Al encontrar niños de 304 años con la capacidad de segmentar palabras en sílabas, mediante canciones o juegos (Vernon, 1997).

El conocimiento silábico permite al niño identificar que las palabras habladas están compuestas por unidades sonoras, a las que se le denomina sílabas o golpes de voz, es a la vez la unidad oral de segmentación más pequeña que es posible articular de manera independiente (Vieiro y Gómez, 2004. Muñoz, 2002).

\section{Conocimiento fonémico}

El conocimiento fonémico es el conocimiento Metalingüístico que permite al niño reconocer que las palabras habladas están compuestas por unidades sonoras discretas, como son los fonemas (Clemente, 2001).

Es comprendido como el nivel más complejo lingüística y cognitivamente, y a diferencia de la sílaba, el fonema requieren de una instrucción específica para que pueda ser desarrollado. Para su adquisición y desarrollo se recomienda ser enseñada de manera sistemática a través de diversas tareas graduadas de segmentación de unidades fonológicas, conteo de sílabas, identificación de unidades iniciales y finales, utilizando material concreto, como fichas e imágenes, que son procedimiento efectivo para el aprendizaje y desarrollo de la conciencia fonológica (Ramos y cuadrados ,2006. Villalón, 2008). 


\section{Enfoque Cognitivo y Psicolingüístico de la lectura}

Se plantea que el aprendizaje lecto-escritor demanda diversas habilidades tanto cognitivas como lingüísticas y psicológicas; entre las cuales predomina la conciencia fonológica, la conciencia silábica, el reconocimiento semántico, la memoria verbal, la abstracción y la categorización verbal. Entre otros como el reconocimiento visual ortográfico de algunas palabras y el conocimiento de algunas letras del alfabeto (Bravo, Villalón y Orellana, 2004).

Bravo (2005) explica tres niveles que ocurren en los niños para aprender a leer y escribir. Teniendo en cuenta que la lectura no se adquiere esporádicamente sino que es un proceso. Estos niveles están clasificado según las demandas cognitivas y lingüísticas que requiera cada uno para su funcionalidad.

Primero, los procesos de bajo nivel; es un procesamiento perceptivo visual que permite observar y extraer los rasgos de las letras y transformarla en códigos, mediante los movimientos oculares de fijación y sacádicos teniendo en cuenta que los signos o letras no están dispuestos aleatoriamente, sino requieren de un desplazamiento espacial, ya sea de izquierda- derecha, arribaabajo. Posteriormente esta información gráfica será almacenada en la memoria sensorial, luego en la memoria de corto plazo y por último en la memoria de largo plazo donde son almacenados los datos más relevantes y convertidos en códigos lingüísticos. En este nivel el niño logra diferenciar un grafema de un dibujo.

Segundo, procesos de nivel medio: En este nivel se transforma las representaciones ortográficas en significados, esto permite que la palabra se pueda reconocer a través de dos vías, una que conecta los signos gráficos con el significado y otra que conecta los signos gráficos en sonido. Lo que es llamado la ruta visual y la ruta fonológica, este nivel trabaja la decodificación, es decir que requiere de una demanda mayor de memoria verbal o memoria de trabajo donde se realiza la decodificación. En la ruta visual o golpe de vista como es denominada, se necesita tener almacenada en la memoria verbal las palabras, para ser decodificada por el niño. En la ruta fonológica es utilizada para decodificar las pseudopalabras o palabras nuevas debido a que requieren del sistema de conversión grafema-fonema.

Tercero, los procesos de alto nivel: En este nivel se asimilan los textos a través de dos procesos: el sintáctico y semántico. El procesamiento sintáctico trabaja todo lo que es la oración, 
tipos de oraciones, signos de puntuación y algunos conectores. El proceso semántico trabaja lo que son conceptos y significados.

Es importante saber que los tres niveles son importantes y se necesitan para lograr un desarrollo adecuado de la conciencia fonológica.

A diferencia de la adquisición del lenguaje oral, el aprendizaje lecto-escritor necesita ser entrenable para ser adquirido como también aquellas habilidades que se requieren para su aprendizaje como la Conciencia Fonológica.

Además diversas investigaciones confirman que existe una relación directa entre la Conciencia Fonológica y el aprendizaje de la lectura y escritura. Demostrando en sus estudios que los niños con un alto nivel de conciencia fonológica eran más sobresalientes que los niños con baja conciencia fonológica en los procesos de aprendizaje lecto-escritor (Griffith y Olson, 1992; Yopp, 1992). No obstante, aunque la conciencia fonológica es catalogada como un predictor necesario para el aprendizaje lector, no es una condición suficiente para decir que los niños con baja conciencia fonológica no aprenderán a leer (Adams, 1990).

En los sistemas de escritura alfabéticos, asignan grafías a los sonidos y los representan de una manera convencional. A lo que se le denomina conversión grafema-fonema. Es aquí donde la conciencia fonológica entra a jugar un papel muy importante, facilitando al hacer consciente a los niños de los diferentes sonidos del lenguaje oral y manipularlos.

\section{Metodología}

\section{Participantes}

La muestra estuvo formada por 48 niños (23 niños y 25 niñas), con edades promedios de 6 años y dos meses con una dispersión de 4 meses aproximadamente, de dos cursos de transición con jornada única en horarios de la mañana. Para el grupo control se tomaron 24 estudiantes y 25 estudiantes para el grupo experimental, siendo la asignación al azar. Dentro de la muestra no habían niños repitentes ni diagnosticados con algún trastorno del aprendizaje.

Tabla 01

Distribución de frecuencia del sexo grupo control y experimental

\begin{tabular}{|c|c|c|c|c|}
\hline \multicolumn{3}{|l|}{ GRUPO } & Frecuencia & Porcentaje \\
\hline Control & Sexo & Femenino & 13 & $54,2 \%$ \\
\hline & & Masculino & 11 & $45,8 \%$ \\
\hline
\end{tabular}




\begin{tabular}{lllll} 
& Total & & 24 & $100 \%$ \\
Experimental & Sexo & Femenino & 12 & $50 \%$ \\
& & Masculino & 12 & $50 \%$ \\
& Total & & 24 & $100 \%$ \\
\hline
\end{tabular}

Fuente propia

Instrumentos

Para comprobar la efectividad del programa "Jugando con los sonidos" se utilizó el test Habilidades Pre-lectoras elaborado por Velarde, Canales, et al. (2013) validado en Perú. Para esta investigación sólo se utilizó el sub-test de la Evaluación de la conciencia fonológica, el cual tiene como finalidad identificar el nivel de reconocimiento y discriminación de rimas, sílabas y fonemas por medio de ítems de administración individual. Se realizaron modificaciones atendiendo a las particularidades apropiadas del lenguaje en el contexto colombiano y se validó por medio de juicio de expertos.

Además se midió su confiabilidad en una muestra piloto de 37 niños colombianos del nivel de transición entre 5,8 años y 6,3 años, de niveles socioeconómicos 1 y 2 (bajo). El índice de confiabilidad de consistencia interna de alfa de chronbach fue de 0,86. Los nombres de las tareas que lo componen son; reconocimiento de sílabas finales, silabas iniciales, sílabas finales, silabas mediales, reconocimiento de fonemas iniciales, fonemas finales, fonemas mediales, Evaluación de Síntesis Fonémica.

\section{Análisis de datos}

En el análisis de los resultados obtenidos en cada uno de los niveles de la conciencia fonológicas, se tomaron los resultados de dos grupos de estudiantes bajo investigación, se analizaron los resultados globales tanto del grupo control y grupo experimental de acuerdo al nivel de conciencia fonológica.

Se realizó un análisis descriptivo por medio de tablas y gráficos donde se tiene en cuenta los resultados de los grupos bajo estudios, para estos se tomaron puntajes totales y se agruparon en rangos teniendo en cuenta el criterio del Subtest de Conciencia Fonológica de la prueba de Habilidades prelectoras de Velarde, Canales et al. (2013). a estos rangos se les da un categorización de acuerdo al alcance de cada nivel, estos resultados se resumen en tablas de 
frecuencia y gráficos de barras. A demás se hizo las respectivas comparaciones de los niveles en el pre y post test.

En el análisis inferencial se aplicó la prueba de U de Mann- Whitney para mirar diferencias entre los puntajes medios de ambos grupos, se eligió esta prueba dado que el Nivel de Rimas no cumplen los supuestos de normalidad para realizar una prueba paramétrica y además por tener tamaños de muestras pequeñas.

\section{Resultados}

\section{Resultados 1}

En el nivel de conciencia fonológica se observa que al inicio del estudio (Pre - Test) tanto en el grupo control como en el experimental presentaban un nivel promedio dado que la gran mayoría de ellos, el $87.5 \%$ de los niños del grupo control y el 95.8\% de los niños que conforman el grupo experimental presenta una conciencia fonológica promedio. Mientras que el 12.5\% del grupo control y el 4.2\% del grupo experimental presentan una conciencia fonológica inferior.

También se logra observa en el Pos -Test que los niños del grupo experimental al que se les aplicó el programa "Jugando con los Sonidos" el 91.7\% alcanzaron un nivel de rimas superior y el $8.3 \%$ están promedio. Mientras la gran mayoría del grupo control el $45.8 \%$ aún se mantienen con un nivel de rimas promedio, sólo el 29.2\% alcanzaron un nivel de rimas superior y el 25\% están en inferior.

\section{Resultados 2}

En el nivel de rima se evidencia que al inicio del estudio (Pre - Test) tanto en el grupo control como en el experimental presentaban un promedio dado que la gran mayoría de ellos, el $50 \%$ de los niños del grupo control y el 54.2\% de los niños que conforman el grupo experimental presenta un nivel de rimas promedio. Mientras que el 41.7\% del grupo control y el 33.3\% del grupo experimental presentan un nivel de rimas inferior. Solamente un $8.3 \%$ del grupo control y un $12.5 \%$ del grupo experimental presentaban un nivel de rimas superior.

Además el Pos -Test los niños del grupo experimental al que se les aplicó el programa "Jugando con los Sonidos" el 91.7\% alcanzaron un nivel de rimas superior y el 8.3\% están 
promedio. Mientras la gran mayoría del grupo control el $45.8 \%$ aún se mantienen con un nivel de rimas promedio, sólo el 29.2\% alcanzaron un nivel de rimas superior y el 25\% están en inferior.

\section{Resultados 3}

En el nivel de Sílaba se observa que al inicio del estudio (Pre - Test) tanto en el grupo control como en el experimental presentaban un nivel promedio dado que la gran mayoría de ellos, el 75\% de los niños del grupo control y el 70.8\% de los niños que conforman el grupo experimental presenta un nivel de sílabas promedio. Mientras que el $25 \%$ del grupo control y el 29.2\% del grupo experimental presentan un nivel de sílabas inferior.

En el Pos -Test que a los niños del grupo experimental al que se le aplicó el programa "Jugando con los Sonidos" el 100\% alcanzaron un nivel de silabas superior. Mientras que la gran mayoría del grupo control el 62.5\% aún se mantienen con un nivel de sílabas promedio, sólo el $12.5 \%$ alcanzaron un nivel de sílabas superior y el $25 \%$ están en inferior.

\section{Resultados 4}

En el nivel de Fonemas se evidencia que al inicio del estudio (Pre - Test) tanto en el grupo control como en el experimental presentaban un nivel promedio dado que el la gran mayoría de ellos, el $66.7 \%$ de los niños del grupo control y el $91.7 \%$ de los niños que conforman el grupo experimental presenta un nivel de fonemas promedio. Mientras que el $25 \%$ del grupo control y el $4.2 \%$ del grupo experimental presentan un nivel de fonemas inferior. . Solamente un $8.3 \%$ del grupo control y un $4.2 \%$ del grupo experimental presentaban un nivel de fonemas superior.

En el Pos - Test que a los niños del grupo experimental al que se le aplicó el programa "Jugando con los Sonidos" el 100\% alcanzaron un nivel de fonemas superior. Mientras que la gran mayoría del grupo control el 62.5\% aún se mantienen con un nivel de fonemas promedio, sólo el 25\% alcanzaron un nivel de fonemas superior y el $12.5 \%$ están en inferior.

En la Tabla 6 se presentan Los resultados de las medidas descriptivas de los puntajes por niveles para el grupo control y experimental tanto en el Pre -Test y Pos - Test

\section{Análisis técnico}

A continuación se presentan la tabla y gráficos de barras de los porcentajes de conciencia fonológica alcanzados por grupos control y experimental en el Pre-Test y Pos-Test. 
Tabla 02

Distribución de frecuencia de conciencia fonológica para grupo control y experimental en el Pre-Test y Pos-Test

\begin{tabular}{lllllll}
\hline Prueba & Grupos & & & Frecuencia & Porcentaje & $\begin{array}{l}\text { Porcentaje } \\
\text { Acumulado }\end{array}$ \\
\hline Pretest & Control & Válidos & Inferior & 3 & 12,25 & 12,5 \\
& & & Promedio & 21 & 87,5 & 100 \\
& \multirow{3}{*}{ Experimental } & \multirow{2}{*}{ Válidos } & Total & 24 & 100 & \\
& & & Inferior & 1 & 4,2 & 4,2 \\
& & & Promedio & 23 & 95,8 & 100 \\
Postest & \multirow{3}{*}{ Control } & Válidos & Inferior & 24 & 100 & \\
& & & Promedio & 18 & 8,3 & 8.3 \\
& & & Superior & 4 & 75,0 & 83,3 \\
& & & Total & 24 & 16,7 & 100 \\
& \multirow{2}{*}{ Experimental } & Válidos & Superior & 24 & 100 & \\
& & & & & 100 & 100 \\
\hline
\end{tabular}

Fuente propia
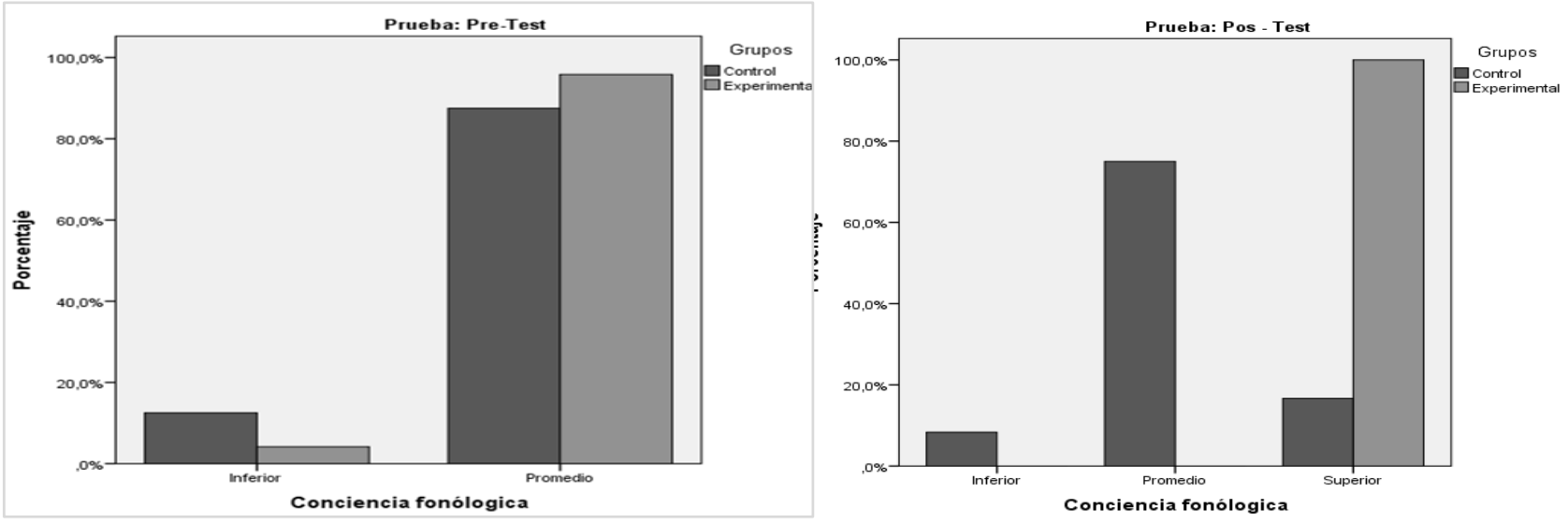

Figura 1. Conciencia fonológica en el Pre - Test para Grupo control y experimental.

Figura 2. Conciencia fonológica en el Pos - Test para Grupo control y experimental

A continuación se presenta las tablas de frecuencia y los gráficos de barras de los porcentajes de nivel de sílaba alcanzados por grupos control y experimental en el Pre-Test y PosTest.

Tabla 03

Distribución de frecuencia del nivel de rimas para grupo control y experimental en el Pre-Test y Pos-Test 


\begin{tabular}{|c|c|c|c|c|c|c|}
\hline Prueba & Grupos & & & Frecuencia & Porcentajes & $\begin{array}{l}\text { Porcentaje } \\
\text { acumulado }\end{array}$ \\
\hline \multirow[t]{8}{*}{ Pretest } & Control & Válidos & Inferior & 10 & 41,7 & 41,7 \\
\hline & & & Promedio & 12 & 50 & 91,7 \\
\hline & & & Superior & 2 & 8,3 & 100 \\
\hline & & & Total & 24 & 100 & \\
\hline & Experimental & Válidos & Inferior & 8 & 33,3 & 33,3 \\
\hline & & & Promedio & 13 & 54,2 & 87,5 \\
\hline & & & Superior & 3 & 12,5 & 100 \\
\hline & & & Total & 24 & 100 & \\
\hline \multirow[t]{7}{*}{ Postest } & Control & Válidos & Inferior & 6 & 25 & 25 \\
\hline & & & Promedio & 11 & 45,8 & 70,8 \\
\hline & & & Superior & 7 & 29,2 & 100 \\
\hline & & & Total & 24 & 100 & \\
\hline & Experimental & Válidos & Promedio & 2 & 8,33 & 8,3 \\
\hline & & & Superior & 22 & 91,7 & 100 \\
\hline & & & Total & 24 & 100 & \\
\hline
\end{tabular}

Fuente propia
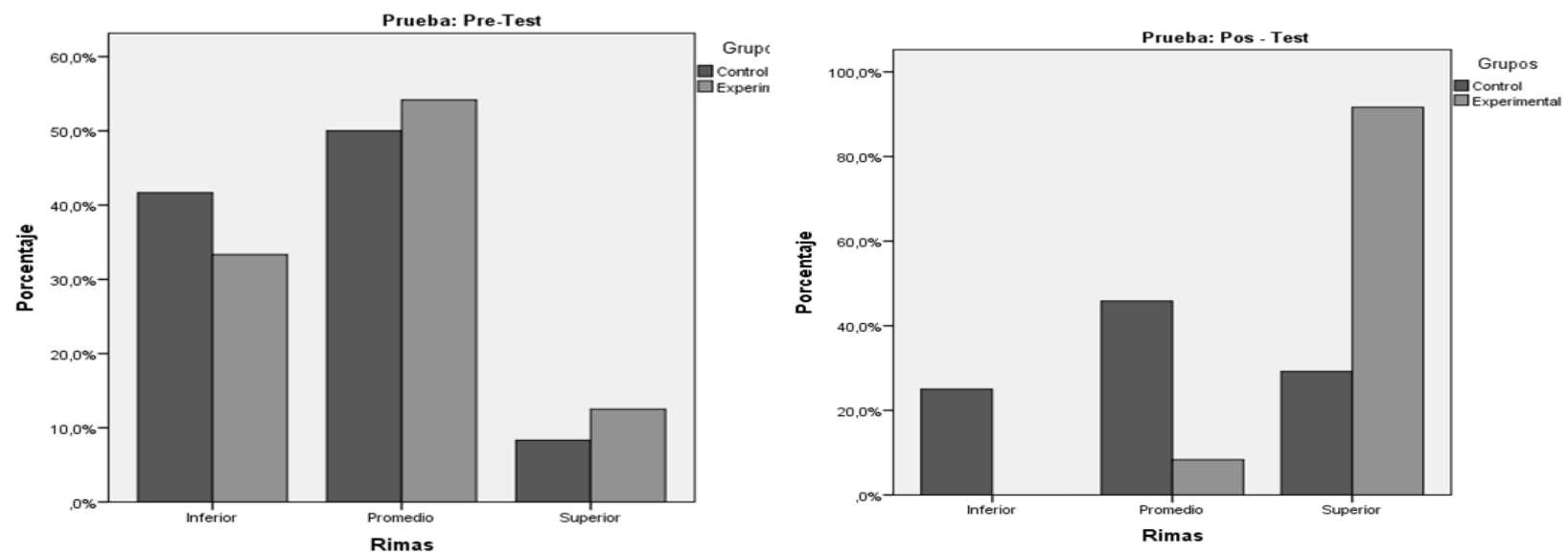

Figura 3. Nivel de Rimas en el Pre - Test para Grupo control y experimental.

Figura 4. Nivel de Rimas en el Pos - Test para Grupo control y experimental

Tabla 04

Distribución de frecuencia de nivel de sílabas para grupo control y experimental en el Pre-Test y Pos-Test

\begin{tabular}{|c|c|c|c|c|c|c|}
\hline Prueba & Grupos & & & Frecuencia & Porcentajes & $\begin{array}{l}\text { Porcentaje } \\
\text { acumulado }\end{array}$ \\
\hline \multirow[t]{6}{*}{ Pretest } & Control & Válidos & Inferior & 6 & 25 & 25 \\
\hline & & & Promedio & 18 & 75 & 100 \\
\hline & & & Total & 24 & 100 & \\
\hline & Experimental & Válidos & Inferior & 7 & 29,2 & 29,2 \\
\hline & & & Promedio & 17 & 70,8 & 100 \\
\hline & & & Total & 24 & 100 & \\
\hline
\end{tabular}




\begin{tabular}{|c|c|c|c|c|c|c|}
\hline \multirow[t]{5}{*}{ Postest } & Control & Válidos & Inferior & 6 & 25 & 25 \\
\hline & & & Promedio & 15 & 62,5 & 87.5 \\
\hline & & & Superior & 3 & 12,5 & 100 \\
\hline & & & Total & 24 & 100 & \\
\hline & Experimental & & Superior & 24 & 100 & 100 \\
\hline
\end{tabular}

Fuente propia

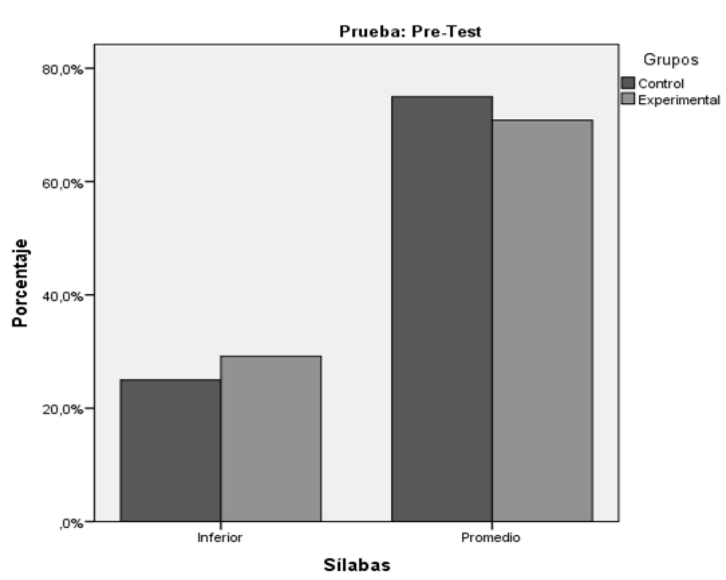

Figura 5. Nivel de Sílabas en el Pre - Test para Grupo control y experimental.

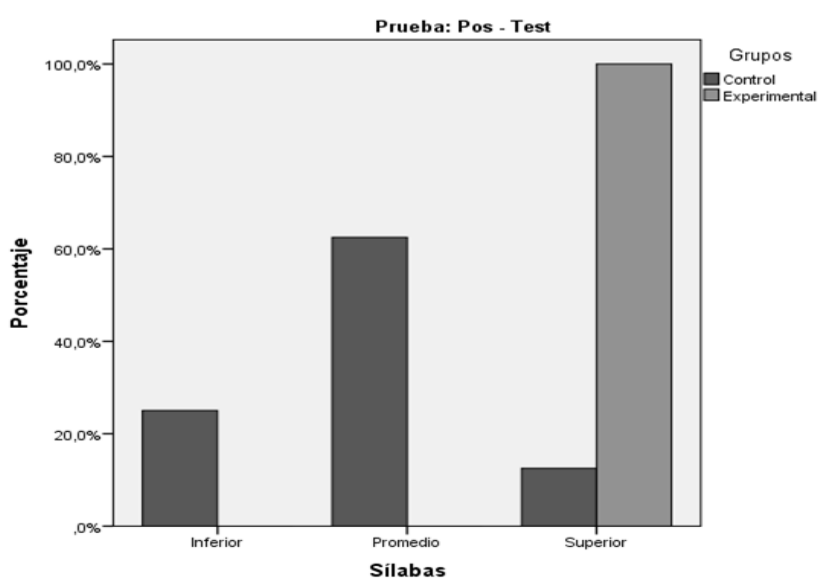

Figura 6. Nivel de Sílabas en el Pos - Test para Grupo control y experimenta I

A continuación se presenta las tablas de frecuencia y los gráficos de barras de los porcentajes de nivel de fonemas alcanzados por grupos control y experimental en el Pre-Test y Pos-Test.

Tabla 05

Distribución de frecuencia del nivel de fonemas para grupo control y experimental en el Pre-Test y Pos-Test

\begin{tabular}{|c|c|c|c|c|c|c|}
\hline Prueba & Grupos & & & Frecuencia & Porcentajes & $\begin{array}{l}\text { Porcentaje } \\
\text { acumulado }\end{array}$ \\
\hline \multirow[t]{8}{*}{ Pretest } & Control & Válidos & Inferior & 6 & 25 & 25 \\
\hline & & & Promedio & 16 & 66,7 & 91.7 \\
\hline & & & Superior & 2 & 8,3 & 100 \\
\hline & & & Total & 24 & 100 & \\
\hline & Experimental & Válidos & Inferior & 1 & 4,2 & 4,2 \\
\hline & & & Promedio & 22 & 91,7 & 95,8 \\
\hline & & & Superior & 1 & 4,2 & 100 \\
\hline & & & Total & 24 & 100 & \\
\hline \multirow[t]{5}{*}{ Pretest } & Control & Válidos & Inferior & 3 & 12,5 & 12,5 \\
\hline & & & Promedio & 15 & 62,5 & 75 \\
\hline & & & Superior & 6 & 25 & 100 \\
\hline & & & Total & 24 & 100 & \\
\hline & Experimental & Válidos & Superior & 24 & 100 & 100 \\
\hline
\end{tabular}


Fuente propia
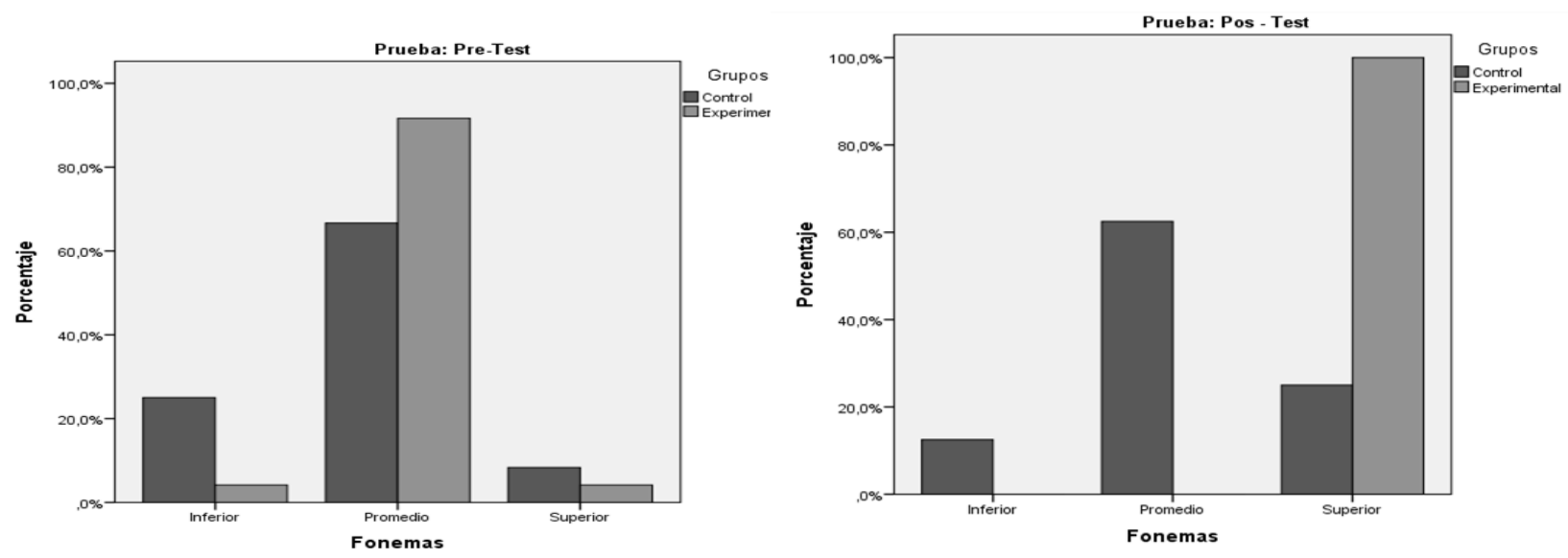

Figura 7. Nivel de fonemas en el Pre - Test para Grupo control y experimental.

Figura 8. Nivel de fonemas en el Pos - Test para Grupo control y experimental

Tabla 06

Análisis de medidas descriptivas de los puntajes alcanzados en el grupo control y experimental en el Pre-Test y Post-Test

\begin{tabular}{|c|c|c|c|c|c|c|}
\hline Prueba & Grupos & & $\mathrm{N}$ & Frecuencia & Porcentajes & $\begin{array}{l}\text { Porcentaje } \\
\text { acumulado }\end{array}$ \\
\hline \multirow[t]{8}{*}{ Pretest } & Control & Rimas & 24 & 4,58 & ,776 & ,601 \\
\hline & & Sílabas & 24 & 9,87 & 1,752 & 3,071 \\
\hline & & Fonemas & 24 & 14,50 & 4,086 & 16,696 \\
\hline & & $\mathrm{N}$ válido(según lista) & 24 & & & \\
\hline & Experimental & Rimas & 24 & 4,71 & ,806 & ,650 \\
\hline & & Sílabas & 24 & 10,25 & 2,507 & 6,283 \\
\hline & & Fonemas & 24 & 15,62 & 2,651 & 7,027 \\
\hline & & $\mathrm{N}$ válido(según lista) & 24 & & & \\
\hline \multirow[t]{8}{*}{ Postest } & Control & Rimas & 24 & 4,71 & 1,367 & 1,868 \\
\hline & & Sílabas & 24 & 12,04 & 3,895 & 15,172 \\
\hline & & Fonemas & 24 & 17,71 & 4,912 & 24,129 \\
\hline & & $\mathrm{N}$ válido(según lista) & 24 & & & \\
\hline & Experimental & Rimas & 24 & 5,92 & ,282 & ,080 \\
\hline & & Sílabas & 24 & 20,71 & 1,488 & 2,216 \\
\hline & & Fonemas & 24 & 29,83 & 2,408 & 5,797 \\
\hline & & $\mathrm{N}$ válido(según lista) & 24 & & & \\
\hline
\end{tabular}

Fuente propia

Se puede observar ver que los puntajes promedio en sílabas, rimas y fonemas en ambos grupos mejoraron en el Post -Test en ambos grupos mejoró en comparación al Pre-Test. Pero el 
promedio del grupo experimental es superior al grupo control después de haber aplicado el programa "A prendiendo con los sonidos" en comparación con el grupo control.

A continuación se realiza la comparación de las media de la conciencia fonológica para el grupo control y experimental del pre - test y post - test.

Tabla 07

Resultados de la prueba $U$ de Mann - Whitney para grupo control y experimental en el Pre - Test y Post-Test

\begin{tabular}{llllll}
\hline \multirow{2}{*}{$\begin{array}{l}\text { Prueba } \\
\text { N }\end{array}$} & $\begin{array}{l}\text { Grupo } \\
\text { Control }\end{array}$ & $\begin{array}{l}\text { Grupo } \\
\text { Experimental }\end{array}$ & $\begin{array}{l}\text { U de Mann- } \\
\text { Whitney }\end{array}$ & $\begin{array}{l}\text { Significancia } \\
\text { (Valor }-\mathrm{p})\end{array}$ \\
\cline { 3 - 5 } & & $\begin{array}{l}\text { Rango } \\
\text { promedio }\end{array}$ & Rango promedio & & \\
\hline Pre - Test & 24 & 22.33 & 26.77 & 233.5 & 0.259 \\
Post - Test & 24 & 12.54 & 36.47 & 1 & $0.000^{* * *}$ \\
\hline$* * *$ Significancia estadística $p<0.01$ & & & \\
\hline
\end{tabular}

Fuente propia

Se puede observar que en el momento de aplicar el pre - Test en ambos grupos su rango promedio es muy similar y de acuerdo a la prueba no paramétrica basada en el estadístico de U de Mann-Whitney se observa que no existe diferencias significativas entre ambos grupos al inicio del estudio dado que el valor $-\mathrm{p}=0.259$ es muy grande (mayor que 0.01). En el Post- Test se logra ver que después de haber aplicado el programa "Jugando con los Sonidos" se puede evidenciar un rango promedio superior en el grupo experimental en comparación al grupo control, esto se reafirma en el momento de aplicar la prueba basada el estadístico de U de Mann-Whitney dado que presenta un Valor $-\mathrm{p}=0.000$ muy pequeño (menor que 0.01 )

En el Post- Test se logra ver que después de haber aplicado el programa "Jugando con los Sonidos" se puede evidenciar un rango promedio superior en el grupo experimental en comparación al grupo control, esto se reafirma en el momento de aplicar la prueba basada el estadístico de $U$ de Mann-Whitney dado que presenta un Valor $-p=0.000$ muy pequeño (menor que 0.01 ) por tanto se puede concluir que la aplicación del programa "Jugando con los Sonidos" es eficaz para lograr los niveles de conciencia fonológica, en los niños del grado de transición de la Institución Cristóbal: Colombia, 2016.

A continuación se realiza una comparación de las media de niveles de conciencia fonológica para el grupo control y experimental del Pre - Test y Post - Test. 
Tabla 08

Resultados de la prueba U de Mann - Whitney por nivel en grupo control y experimental en el Pre - Test y Post - Test

\begin{tabular}{|c|c|c|c|c|c|c|}
\hline \multirow[t]{2}{*}{ Nivel } & \multirow{2}{*}{ Prueba } & \multirow{2}{*}{$\mathrm{N}$} & $\begin{array}{l}\text { Grupo } \\
\text { Control }\end{array}$ & $\begin{array}{l}\text { Grupo } \\
\text { Experimental }\end{array}$ & \multirow{2}{*}{$\begin{array}{l}\text { U de } \\
\text { Mann- } \\
\text { Whitney }\end{array}$} & \multirow{2}{*}{$\begin{array}{l}\text { Significancia } \\
\text { (Valor }-p)\end{array}$} \\
\hline & & & Rango promedio & Rango promedio & & \\
\hline \multirow[t]{2}{*}{ Rima } & Pre - Test & 24 & 23.38 & 25.63 & 261 & 0.542 \\
\hline & Post - Test & 24 & 16.75 & 32.25 & 102 & 0.000 \\
\hline \multirow[t]{2}{*}{ Fonemas } & Pre - Test & 24 & 21.23 & 27.77 & 209.5 & 0.104 \\
\hline & Post - Test & 24 & 12.75 & 36.25 & 6.000 & 0.000 \\
\hline \multirow[t]{2}{*}{ Sílabas } & Pre - Test & 24 & 23.44 & 25.56 & 262.5 & 0.595 \\
\hline & Post - Test & 24 & 13.06 & 35.94 & 12.5 & 0.000 \\
\hline
\end{tabular}

Fuente propia

En el post - test se logra evidenciar después de haber aplicado el programa "Jugando con los Sonidos" que el rango promedio es superior en el grupo experimental en comparación al grupo control, esto se reafirma en el momento de aplicar la prueba basada el estadístico de U de MannWhitney dado que presenta un Valor $-\mathrm{p}=0.000$ muy pequeño (menor que .05 ), por ende e puede concluir que la aplicación del programa "Jugando con los Sonidos" es eficaz para lograr los niveles de rima, sílaba y Fonemas en los niños del grado de transición de la Institución Cristóbal: Colombia, 2016

\section{Conclusión}

El Programa "Jugando con los sonidos" es eficaz para incrementar los niveles de la conciencia fonológica en niños del nivel de transición de una institución educativa colombiana, al aplicar la prueba basada en el estadístico de $U$ de MannWhitney donde presenta un Valor $-\mathrm{p}=0.000$ muy pequeño (menor que 0.05).

\section{Referencias}

Adam, M. (1990). Beginning to read: Thinking and learning about print., Cambridge: MIT Press.

Alsina, L., León, A., Pino, M. (2011). Conciencia Fonológica y su relaión con las dificultades de la lectura. Cultura, Educación y Sociedad. p. 25 - 33. Recuperada de 
http://revistascientificas.cuc.edu.co/index.php/culturaeducacionysociedad/article/viewFile/929 /pdf_150

Anthony, J. L., \& Francis, D. J. (2005). Development of Phonological Awareness. Current Directions in Psychological Science, 15 (5). p. 255-259. Recuperado de http://citeseerx.ist.psu.edu/viewdoc/download?doi=10.1.1.525.5413\&rep=rep1\&type=pdf.

Arancibia, B., Bimaza, M., Saenz, K. (2012). Aplicación de un Programa de Estimulación en conciencia fonolológica en preescoalres de nivel de transición 2 y alumnos de primer año básico pertenecientes a escuelas vulnerables de Provincias de Concepción. Revista Signos , vol. 45 (80), p. 236 - 256. Recuperado de http://www.scielo.cl/scielo.php?script=sci_arttext\&pid=S0718-09342012000300001.

Ardila, A., Rosselli, M., Matute, E. (2005). Neuropsicología de los Transtornos del aprendizaje, México: Manual Moderno.

Bernardi, C., Mishima, F., Dos Santos, P (2013) Treinamento breve de consciência fonológica: impacto sobre a alfabetização. Revista De Psicopedagogía. 30 (93). p. 189- 200. Recuperado de http://pepsic.bvsalud.org/pdf/psicoped/v30n93/05.pdf.

Bos, M., Gaminian, A., Veggmentaa, E. (2014). América Latina en PISA 2012, BIF $n^{\circ} 11$; Chile en PISA 2012 Logros y desafíos Pendientes. Recuperado de http://scholar.harvard.edu/files/alejandro_ganimian/files/brief_11.pdfm=1407191003

Bravo, L (2005) Lenguaje y dislexia. Enfoque cognitivo del retardo lector. Santiago de Chile: Universidad Católica de Chile.

Bravo, L. Villalón, M. Orellana, E (2004). Procesos Cognitivos y el aprendizaje de la lectura inicial: diferencias cognitivas entre buenos lectores y lectores deficientes. Estudios Pedagógicos. 30, p. 7 - 19. Recuperado de http://www.scielo.cl/scielo.php?script=sci_arttext\&pid=S0718-07052004000100001

Bustamante, N. (2015) Niños colombianos pasan raspando en habilidades lectoras tomado de http://www.eltiempo.com/estilo-de-vida/educacion/comprensión-de-lectura-de-losestudiantes-colombianos-/15283357

Caman, L., Velarde, E. (2010) Programa Jugando con los sonidos y niveles de la conciencia fonológica en niños de cinco años de una institución educativa. Universidad San Ignacio de Loyola. Recuperado de http://repositorio.usil.edu.pe/bitstream/123456789/1102/1/2010

Cadavid, N., Quijano, C., Tenorio, M., Rosas, R. (2014) El juego como vehículo para mejorar las habilidades de la lectura en niños con dificultades lectora. Pensamiento Psicológico. 12 (1), p. $23-38$.

Cardona, M., Cadavid, N (2013) Perfil lector de niños con y sin retraso lector en la ciudad de Cali (Colombia). Psicología desde el Caribe. 30 (2) Recuperado de http://www.scielo.org.co/scielo.php?pid=S0123-417X2013000200004.

Carrillo, M., Gallego, J (1992). Desarrollo metafonológico y adquisición de la lectura; Un programa de entrenamiento. Ministerio de Educación y Ciencia. CIDE. España

Cortés, C,. Flórez, R., Goméz, D (2016). Propuesta de inserción de algunos principios del pensamiento complejo en la enseñanza y el aprendizaje de la lectura y la escritura en grado 
primero. Repositorio Universidad Nacional de Colombia. Recuperado de http://www.bdigital.unal.edu.co/53156/1/52753284.2016.pdf.

Fernández, L (2002) Niveles de pobreza y desarrollo socioeconómico en América Convergencia. Revista de Ciencias Sociales, 9 (28). p. 0

Flórez, R., Restrepo, M., Schwanenflugel, P (2009) Promoción del alfabetismo inicial y prevención de las dificltades en lalectura: una experiencia pedagógica en el aula de preescolar. Avances en Psicología Latinoamericana.27 (1). p. 79 -96. Recuperada de http://www.redalyc.org/pdf/799/79911627006.pdf

Gallego, J., Angustias, I., Gómez, M. (2015). Trastorno Fonológico en niños; resultado de la aplicación de un programa. Trastorno Fonológico en niños; resultado de la aplicación de un programa. Revista de Investigación Educativa. 33 (2) Recuperado de http://www.redalyc.org/articulo.oa?id=283341409013

Griffith, P., Olson M. (1992) Phonemic Awareness Helps Beginning Readers Break the Code. The Reading Teacher. 45(7). pp. 526 - 523. Recuperado de www.jstor.org/stable/20200912

Guarneros, E., Vega, L (2014). Habilidades Lingüísticas orales y escritas para la lectura y la escritura en niños preescolares. 32(1). p. 25 - 31. Doi: dx.doi.org/10.12804/ap132.1.2014.02

Gutiérrez, R., Diez, A. (2017) Efectos de un programa de Conciencia Fonológica en el Aprendizaje de la Lectura y Escritura. Revista de Reorientación y Psicología. 28 (2). p. 30 45. Recuperada HabilidadesLinguisticasEnNinosDeEstratoSociocultur-2524745\%20(1).pdf

Jager, M., Foorman. B., Lundberg, I., Beeleer, T (1998). Phonemic Awareness in Young Children. United States: Brookes Publishing Co.

Meneses, A., Garzón, M., Macias, J. (2012) Intervención en Conciencia Fonológica, en el aula para niños de primer ciclo. Revista Cuadernos Hispanoamericanos de Psicología. 12 (2). p. 65 - 79. Recuperado de http://m.uelbosque.edu.co/sites/default/files/publicaciones/revistas/cuadernos hispanoamerica nos_psicologia/volumen12_numero2/articulo_6.pdf

Santiuste, V., López, C. (2004). Nuevos aportes a la intervención en las dificultades de la lectura. Universitas Psychologicas, 4 (1), p. 13 - 22. Recuperado de http://www.redalyc.org/articulo.oa? $\mathrm{id}=64740102$

Ramos, J., Cuadrado, I. (2006). Manual para la evaluación del conocimiento fonológico, Madird.,España: Editorial EOS.

Rosenberg, C., Stein, A. (2015) Análisis Longitudinal del impacto de un programa de alfabetización temprana. Revista Latinoamericana, ciencia, sociedad, niñez y juventud. 14 (2). Recuperado de http://www.scielo.org.co/pdf/rlcs/v14n2/v14n2a15.pdf

Varela, K., Viecco, S., Flórez., S (2014) Diseño y ejecución de actividades para la estimulación en conciencia fonológica en los niveles de Prejardín y Jardín. Zona Próxima. p. 49 - 58. Recuperada de http://www.scielo.org.co/pdf/zop/n20/n20a06.pdf

Vernon, S. (1998) Escritura y Conciencia Fonológica en Niños Hispanoparlantes. Infancia y Aprendizaje. 81.p. $105-120$ 
Velarde, E. (2008). Elaboración y aplicación de un programa metafonológico en niños de 8 a 10 años de tercero y cuarto grado de primaria del cercado de Callao. Investigación Educativa, 14 (25). pp 137 - 154. Recuperado de

http://sisbib.unmsm.edu.pe/bibvirtual/publicaciones/inv_educativa/2010_n25/pdf/a08v14n25. pdf

Vieiro, I., Gómez, V. (2004). Psicología de la lectura: procesos, teoría y aplicaciones instruccionales. Madrid: Pearson.

Yopp, H., and Key (1992). Developing phonemic awareness in young children. The Reading Teacher 45 (9), p. 696-703. Recuperado de teracyhow.com/wpcontent/uploads/2013/08/SupportingPhonemicAwarenessDevelopmentintheClassroom.pdf 